\title{
A CONVERGENCE THEOREM FOR RIEMANNIAN SUBMANIFOLDS
}

\author{
ZHONGMIN SHEN
}

\begin{abstract}
In this paper we study the convergence of Riemannian submanifolds. In particular, we prove that any sequence of closed submanifolds with bounded normal curvature and volume in a closed Riemannian manifold subconverge to a closed submanifold in the $C^{1, \alpha}$ topology. We also obtain some applications to irreducible homogeneous manifolds and pseudo-holomorphic curves in symplectic manifolds.
\end{abstract}

\section{INTRODUCTION}

Let $\mathscr{M}(n, K, v, D)$ be the class of manifolds $(M, g)$ satisfying the bounds

$$
|\sec (M)| \leq K, \quad \operatorname{vol}(M) \geq v, \quad \operatorname{diam}(M) \leq D .
$$

The well-known convergence theorem says that given a sequence of manifolds $\left(M_{i}, g_{i}\right) \in \mathscr{M}(n, K, v, D)$, there is a subsequence, $\left(M_{i^{\prime}}, g_{i^{\prime}}\right)$, a $C^{1, \alpha}$ Riemannian $n$-manifold $\left(M_{0}, g_{0}\right)$, and $C^{2, \alpha}$ diffeomorphisms $\phi_{i^{\prime}}: M_{0} \rightarrow M_{i^{\prime}}$, such that $\phi_{i^{\prime}}^{*} g_{i^{\prime}}$ converges to $g_{0}$ in the $C^{1, \alpha^{\prime}}$ topology $\left(\alpha^{\prime}<\alpha\right)$. We refer to, for example, [Ch, N1, N2, G1, Ps, GW, K] for details. M. Anderson has extended this theorem to a larger class of Riemannian manifolds (see [AM]).

In this paper we are going to establish a convergence theorem for Riemannian submanifolds. Fix a complete Riemannian $m$-manifold $(\widetilde{M}, \tilde{g})$. We will denote by $(M, f)$ an $n$-dimensional submanifold in $(\widetilde{M}, \tilde{g})$, where $M$ is a smooth $n$-manifold and $f$ is an immersion from $M$ into $\widetilde{M}$. One has the natural orthogonal decomposition $T_{f(x)} \widetilde{M}=f_{*}\left(T_{x} M\right) \oplus T_{x} M^{\perp}$. The normal curvature $I I_{f}: T_{x} M \otimes T_{x} M \rightarrow T_{x} M^{\perp}$ is defined by the formula

$$
I I_{f}(u, v)=-\left(\tilde{\nabla}_{U} V\right)^{\perp},
$$

where $U$ and $V$ are local extensions of $f_{*}(u)$ and $f_{*}(v)$ on $\widetilde{M}$ and $\widetilde{\nabla}$ denotes the Levi-Civita connection of $\tilde{g}$. Throughout this paper we will always denote by $\sec (f), \operatorname{scal}(f), \operatorname{inj}(f)$, and $\operatorname{vol}(f)$ the sectional curvature, the scalar curvature, the injectivity radius, and the volume of $\left(M, f^{*} \tilde{g}\right)$, respectively.

Received by the editors October 20, 1993 and, in revised form, December 16, 1993; originally communicated to the Proceedings of the AMS by Christopher Croke.

1991 Mathematics Subject Classification. Primary 53C20, 53C40; Secondary 53C15, 53C30.

Key words and phrases. Riemannian submanifold, convergence in the $C^{1, \alpha}$ topology, normal curvature, volume, isoperimetric inequality, injectivity radius, pseudo-holomorphic curve, homogeneous manifold. 
Let $\mathscr{S}_{n}(\Lambda, V)$ denote the class of $n$-dimensional closed submanifolds ( $M$, $f$ ) in $\widetilde{M}$, which satisfy the bounds

$$
\left|I I_{f}\right| \leq \Lambda, \quad \operatorname{vol}(f) \leq V .
$$

We have the following

Theorem 1. Given any uniformly bounded sequence $\left(M_{i}, f_{i}\right) \in \mathscr{S}_{n}(\Lambda, V)$, namely, $\bigcup_{i=1}^{\infty} f_{i}\left(M_{i}\right)$ is contained in a compact subset of $\widetilde{M}$. There is a subsequence $\left(M_{i^{\prime}}, g_{i^{\prime}}\right)$, a $C^{1, \alpha}$ Riemannian manifold $\left(M_{0}, g_{0}\right)$, and $C^{2, \alpha}$ diffeomorphisms $\phi_{i^{\prime}}: M_{0} \rightarrow M_{i^{\prime}}$, such that $f_{i^{\prime}} \circ \phi_{i^{\prime}}$ converges to a $C^{1, \alpha}$ immersion $f_{0}$ in the $C^{1, \alpha^{\prime}}$ topology, for any $\alpha^{\prime}<\alpha$, with $f_{0}^{*} \tilde{g}=g_{0}$.

If in addition $\operatorname{inj}\left(f_{i}\right) \geq i_{0}>0$ for all $i$, the assertion of Theorem 1 follows immediately, see $\S 3$ or [K]. It is well known that for a Riemannian manifold, the sectional curvature bound does not give a lower bound on the injectivity radius. A flat torus is a simple example. For a Riemannian submanifold $(M, f)$, contrary to the sectional curvature case, the normal curvature bound $\left|I I_{f}\right| \leq \Lambda$ does imply $\operatorname{inj}(f) \geq i_{0}$ for some number $i_{0}$ depending on $\Lambda$ and the ambient space. This fact is somewhat surprising. In [AL] L. Andersson has given such a lower bound on $\operatorname{inj}(f)$ when the ambient space is Euclidean. Then, using results by Cheeger $[\mathrm{Ch}]$ and Andersson $[\mathrm{AL}], \mathrm{R}$. Howard $[\mathrm{H}]$ asserts that there are only finitely many diffeomorphism types among $n$-dimensional closed Riemannian submanifolds $(M, f)$ in $\mathbb{R}^{m}$ with $\left|I I_{f}\right| \leq \Lambda$ and $\operatorname{vol}(f) \leq V$.

The main idea of Theorem 1 is to prove a priori estimates on the injectivity radius for Riemannian submanifolds in a general Riemannian manifold. Our treatment is quite different from Andersson's.

Theorem 1 has a number of applications. For a closed submanifold $(M, f)$ in $(\widetilde{M}, \tilde{g})$, we denote by $\operatorname{tr} I I_{f}$ the mean curvature of $f$, which is defined by

$$
\operatorname{tr} I I_{f}=\sum_{i} I I_{f}\left(e_{i}, e_{i}\right),
$$

where $\left\{e_{i}\right\}$ is an orthonormal basis on $\left(M, f^{*} \tilde{g}\right)$. Suppose that $(M, f)$ satisfies the bounds

$$
\left|\operatorname{tr} I I_{f}\right| \leq H, \quad \operatorname{scal}(f) \geq-\lambda, \quad \operatorname{vol}(f) \leq V .
$$

Further, suppose that the sectional curvature of $\widetilde{M}$ is bounded from above by $K$ along $f(M)$. Then it follows from the Gauss equation that $\left|I I_{f}\right| \leq$ $\Lambda(n, K, H, \lambda)$.

Let $\mathscr{V}_{n}(H, \lambda, V)$ be the class of all $n$-dimensional closed submanifolds $(M, f)$ in $(\widetilde{M}, \tilde{g})$, which satisfy $(3)$. We have the following

Corollary 1. Given positive numbers $H, \lambda$, and $V$, the class $\mathscr{V}_{n}(H, \lambda, V)$ is precompact in the $C^{1, \alpha}$ topology $(\alpha<1)$ in the sense of Theorem 1 .

Remark. The lower bound on the scalar curvature in Corollary 1 is necessary even when $n=2$. See [G2] for some examples.

Let $(N, \omega)$ be a closed symplectic manifold with an $\omega$-tamed almost complex structure $J$, i.e.,

$$
\omega(v, J v)>0, \quad v \neq 0 .
$$


Then there is a naturally induced Hermitian metric $g_{J}$ defined by

$$
g_{J}(u, v)=\frac{1}{2}(\omega(u, J v)+\omega(v, J u)) .
$$

Let $(\Sigma, j)$ be a closed Riemannian surface, where $j$ is a complex structure $j$, and $f: \Sigma \rightarrow N$ be a pseudo-holomorphic map, i.e., $d f \circ j=J \circ d f$. It is well known that $f$ has isolated branch points ( $p$ is called a branch point of $f$ if $d f_{p}$ is not injective, otherwise $p$ is called a regular point). It was proved in [G2] that at regular points of $f$,

$$
\left|\operatorname{tr} I I_{f}\right| \leq C_{1},
$$

where $C_{1}$ is a constant independent of $f$. Further if $f$ represents a class $\alpha \in H_{2}(N, \mathbb{Z})$, then

$$
\operatorname{vol}(f) \leq V
$$

where $V$ is a constant independent of representatives $f$ in $\alpha$.

By the Gauss equation, $\operatorname{scal}(f) \leq C_{2}$, where $C_{2}$ is also independent of $f$. However, there is no lower bound on the $\operatorname{scal}(f)$. Gromov's compactness theorem [G2] says that any sequence of (regular) pseudo-holomorphic curves $\left(\Sigma, f_{i}\right)$ satisfying (5), subconverges to a cusp-curve (see also [PW, Y]). In particular, the limit may have singularities. Our next corollary asserts that there is no singular point in the limit if in addition $\operatorname{scal}\left(f_{i}\right) \geq-\lambda$ for all $i$. This property follows from Corollary 1 and (4). More precisely, we have the following

Corollary 2. Let $(\Sigma, j)$ and $(N, \omega, J)$ be as above. Let $f_{i}: \Sigma \rightarrow N$ be a sequence of regular $J$-holomorphic maps satisfying

$$
\operatorname{scal}\left(f_{i}\right) \geq-\lambda, \quad \operatorname{vol}\left(f_{i}\right) \leq V .
$$

Then there exists a sequence of diffeomorphisms $\phi_{i^{\prime}}$ of $\Sigma$ such that $f_{i^{\prime}} \circ \phi_{i^{\prime}}$ converges to a $C^{1, \alpha}$ regular pseudo-holomorphic map $f_{0}$.

We shall apply Theorem 1 to a certain class of homogeneous Riemannian manifolds.

Proposition 1. Given positive numbers $\lambda, v$, and $D$, let $\mathscr{H}_{n}(\lambda)$ denote the class of irreducible homogeneous Riemannian $n$-manifolds $(M, g)$ which satisfy the bounds

$$
\operatorname{scal}(M) \geq-\lambda, \quad \operatorname{diam}(M)=1 .
$$

Then $\mathscr{H}_{n}(\lambda)$ is compact in the $C^{\infty}$ topology. In particular, $\mathscr{H}_{n}(\lambda)$ contains finitely diffeomorphism types.

The proof of Proposition 1 will be given in the last section. It is not known to the author whether there are only finitely many diffeomorphism types among irreducible homogeneous Riemannian manifolds in each dimension.

Acknowledgments. I would like to thank R. Howard for his important comments. Thanks also to Y. Ruan for many discussions on pseudo-holomorphic curves in symplectic manifolds.

\section{ISOPERIMETRIC INEQUALITIES}

Let $(M, g)$ be a complete Riemannian $n$-manifold. For any positive number $\mu>0$, define the $\mu$-isoperimetric constant of $(M, g)$ by the formula

$$
I_{\mu}(M)=\inf \left\{\frac{\operatorname{vol}(\partial \Omega)}{\operatorname{vol}(\Omega)^{(n-1) / n}}, \operatorname{vol}(\Omega) \leq \mu\right\} .
$$


Put $I(M)=I_{\mu}(M)$ for $\mu=\frac{1}{2} \operatorname{vol}(M)$. This is the so-called isoperimetric constant of $(M, g)$.

It is easy to see that for a closed Riemannian $n$-manifold $M$ with $I_{\mu}(M)>$ $0, \operatorname{vol}(M)>\mu$. Furthermore one has the following

Lemma 1. Let $(M, g)$ be a complete Riemannian n-manifold. Suppose that $I_{\mu}(M) \geq I_{0}>0$ for some $\mu>0$. Then for all $r \leq r_{0}$, where $r_{0}=n I_{0}^{-1} \mu^{1 / n}$,

$$
\operatorname{vol}(B(p, r)) \geq n^{-n} I_{0}^{n} r^{n}
$$

Proof. First note that $I_{\mu}(M) \geq I_{0}$ is equivalent to the inequality

$$
\operatorname{vol}(\partial \Omega) \geq I_{0} \operatorname{vol}(\Omega)^{\frac{n-1}{n}}
$$

for all domains $\Omega$ with $\operatorname{vol}(\Omega) \leq \mu$.

Let $B(p, r)$ be a metric ball in $M$ with radius $r \leq r_{0}$. First we suppose that $\operatorname{vol}(B(p, r))>\mu$. Then

$$
\operatorname{vol}(B(p, r))>n^{-n} I_{0} r_{0}^{n} \geq n^{-n} I_{0}^{n} r^{n} .
$$

Suppose that $\operatorname{vol}(B(p, r)) \leq \mu$. Then (7) implies the function $f(t):=$ $\operatorname{vol}(B(p, t))$ satisfies

$$
f^{\prime}(t) \geq I_{0} f(t)^{(n-1) / n}, \quad t \leq r .
$$

It follows from the above inequality that $f(t) \geq n^{-n} I_{0}^{n} t^{n}, t \leq r$. In particular, one obtains

$$
\operatorname{vol}(B(p, r))=f(r) \geq n^{-n} I_{0}^{n} r^{n} .
$$

In order to estimate the injectivity radius we need the following

Lemma 2 [CGT, Theorem 4.3]. Let $(M, g)$ be a complete $n$-manifold with $H \leq \sec (M) \leq K$. Suppose that at a point $p \in M$,

$$
\operatorname{vol}(B(p, s)) \geq c_{0}
$$

for some $s \leq \pi / 4 \sqrt{K}$. Then the injectivity radius at $p$ satisfies

$$
\operatorname{inj}(p) \geq \frac{1}{1+V^{H}(3 s) / c_{0}} \cdot s,
$$

where $V^{H}(r)$ denotes the volume of $r$-ball in the space form of constant curvature $H$.

Corollary 3. Suppose that $(M, g)$ is a complete Riemannian n-manifold satisfying the bounds

Then

$$
I_{\mu}(M) \geq I_{0}, \quad|\sec (M)| \leq K .
$$

$$
\operatorname{inj}(M) \geq c\left(n, \mu, I_{0}, K\right)>0 .
$$

Proof. This follows from Lemmas 1 and 2.

Let $(M, g)$ be a closed Riemannian $n$-manifold, and let $C_{M}(\varepsilon)$ denote the maximal number of disjoint metric $\varepsilon / 2$-balls in $M$. Suppose that $M$ satisfies the bounds

$$
I_{\mu}(M) \geq I_{0}, \quad \operatorname{vol}(M) \leq V .
$$

Then it follows from Lemma 1 that for all $\varepsilon \leq \varepsilon\left(n, I_{0}, \mu\right)$

$$
C_{M}(\varepsilon) \leq C\left(n, I_{0}, V\right) \varepsilon^{-n}
$$

Let $\mathscr{C}\left(n, \mu, I_{0}, V\right)$ denote the class of all closed $n$-manifolds satisfying (8). The following proposition follows from (9) and [G1]. 
Proposition 2. $\mathscr{C}\left(n, \mu, I_{0}, V\right)$ is pre-compact in the Gromov-Hausdorff topology.

From now on we will only consider immersions in a complete Riemannian $m$-manifold $(\widetilde{M}, \tilde{g})$. Suppose that $f$ is complete, i.e., the pull-back metric $f^{*} \tilde{g}$ is complete, and it has bounded mean curvature. Then $\left(M, f^{*} \tilde{g}\right)$ has positive $\mu$-isoperimetric constant for some $\mu>0$, provided that $\widetilde{M}$ has bounded geometry along the image of $f$. Let $\widetilde{U}_{f}=\{y \in \widetilde{M}$, $\operatorname{dist}(y, f(M))<1\}$, and $I_{\mu}(f)=I_{\mu}\left(M, f^{*} \tilde{g}\right)$.

Theorem 2 (Hoffman and Spruck [HS]). Suppose that

$$
\sec \left(\widetilde{U}_{f}\right) \leq K, \quad \operatorname{inj}\left(\widetilde{U}_{f}\right) \geq i_{0}, \quad\left|\operatorname{tr} I I_{f}\right| \leq \Lambda .
$$

Then there are constants $c(n)>0$ and $\mu=\mu\left(n, K, i_{0}, \Lambda\right)>0$, such that

$$
I_{\mu}(f) \geq c(n) \text {. }
$$

Remark. This type of theorem in the case of $\widetilde{M}=\mathbb{R}^{m}$ was first obtained by J. Michael and L. Simon [MS].

Proposition 3. Suppose that

$$
\left|\sec \left(\widetilde{U}_{f}\right)\right| \leq K, \quad \operatorname{inj}\left(\widetilde{U}_{f}\right) \geq i_{0}, \quad\left|I I_{f}\right| \leq \Lambda .
$$

Then there is a constant $c\left(n, K, i_{0}, \Lambda\right)>0$ such that

$$
\operatorname{inj}(f) \geq c\left(n, K, i_{0}, \Lambda\right) .
$$

Proof. By Theorem 2 one has $I_{\mu}(f) \geq c(n)$ for some $\mu=\mu\left(n, K, i_{0}, \Lambda\right)>$ 0 . Then Proposition 3 follows from the Gauss equation and Corollary 3.

Remark. Proposition 3 generalizes a result of L. Andersson who treated the case when the ambient space is Euclidean. Our treatment, however, is quite different from his.

\section{Proof of TheOREM 1}

Before going into proof, we shall recall first some basic facts on harmonic coordinates. We refer the reader to $[\mathrm{JK}]$ and $[\mathrm{GW}]$ for details.

A coordinate $\mathbf{x}=\left(x^{A}\right): U \subset \widetilde{M} \rightarrow \mathbb{R}^{m}$ is said to be harmonic if $\Delta_{\tilde{g}} x^{A}=0$, $1 \leq A \leq m$. We suppose that $(\widetilde{M}, \tilde{g})$ satisfies the bounds

$$
|\sec (\widetilde{M})| \leq K, \quad \operatorname{inj}(\widetilde{M}) \geq i_{0} .
$$

Then there is a number $\delta\left(m, K, i_{0}\right)>0$ such that given any metric ball $B_{\delta}(p)$, $\delta<\delta\left(m, K, i_{0}\right)$, there is a harmonic coordinate $\mathbf{x}=\left(x^{A}\right)$ on $B_{\delta}(p)$ which has the following properties:

(i) $\left(1+\eta_{0}(m, K \delta)\right)^{-1} \delta_{A B} \leq g_{A B} \leq\left(1+\eta_{0}(m, K \delta)\right) \delta_{A B}$, where $g_{A B}=$ $\tilde{g}\left(\partial_{A}, \partial_{B}\right)$;

(ii) $\left\|g_{A B}\right\|_{C^{1, \alpha}\left(B_{\delta}(p)\right)} \leq \eta_{1}\left(m, K, i_{0}, \alpha\right)$; and

(iii) $\left\|x^{A}\right\|_{C^{2, \alpha}\left(B_{\delta / 2}(p)\right)} \leq \eta_{2}\left(m, K, i_{0}, \alpha\right)\left\|x^{A}\right\|_{C^{0}\left(B_{\delta}(p)\right)}$.

Remark. Using the idea of [AL] and the above properties for harmonic coordinates, one can also prove a priori estimates on the injectivity radius for Riemannian submanifolds. 
Now we start to prove Theorem 1. Given any sequence of immersions $\left(M_{i}, f_{i}\right) \in \mathscr{S}_{n}(\Lambda, V)$. By assumption we may assume that $\bigcup_{i} f_{i}\left(M_{i}\right) \subset F$ for some compact subset $F$ in $\widetilde{M}$. Let $\widetilde{U}_{F}=\{\tilde{x} \in \widetilde{M}$, $\operatorname{dist}(\tilde{x}, F)<1\}$. Suppose that

$$
\left|\sec \left(\widetilde{U}_{F}\right)\right| \leq K, \quad \operatorname{inj}\left(\widetilde{U}_{F}\right) \geq i_{0} .
$$

By Proposition 3 and the Gauss equation, one has

$$
\left|\sec \left(f_{i}\right)\right| \leq K+2 \Lambda^{2}, \quad \operatorname{inj}\left(f_{i}\right) \geq c\left(n, K, \Lambda, i_{0}\right) .
$$

Put $g_{i}=f_{i}^{*} \tilde{g}$. Consider the sequence of Riemannian manifolds $\left(M_{i}, g_{i}\right)$. By Gromov's compactness theorem (see, e.g., [K, AM]), there exist a subsequence $\left(M_{k}, g_{k}\right)$, a closed $C^{1, \alpha}$ Riemannian $n$-manifold $\left(M_{0}, g_{0}\right)$, and $C^{2, \alpha}$ diffeomorphisms $\phi_{k}: M_{0} \rightarrow M_{k}$ such that $\tilde{g}_{k}:=\phi_{k}^{*} g_{k}$ converges to $g_{0}$ in the $C^{1, \alpha^{\prime}}$ topology $\left(\alpha^{\prime}<\alpha\right)$. Fix a harmonic coordinate system $\left(x^{A}\right)$ on $(\widetilde{M}, \tilde{g})$ and a harmonic coordinate system $\left(x^{a}\right)$ on $\left(M_{0}, g_{0}\right)$. Put $\tilde{f}_{k}=f_{k} \circ \phi_{k}$. We have

$$
\left(\tilde{g}_{k}\right)_{a b}:=\partial_{a} \tilde{f}_{k}^{A} \partial_{b} \tilde{f}_{k}^{B} g_{A B} \rightarrow g_{a b},
$$

in the $C^{1, \alpha^{\prime}}$ topology $\left(\alpha^{\prime}<\alpha\right)$, where $g_{A B}=\tilde{g}\left(\partial_{A}, \partial_{B}\right), g_{a b}=g_{0}\left(\partial_{a}, \partial_{b}\right)$. Thus one obtains a uniform $C^{0}$ bound on $\partial_{a} \tilde{f}_{k}$. Let $I I_{k}=I I_{\tilde{f}_{k}}$. By the definition of $I I_{k}$, one has

$$
\partial_{a} \partial_{b} \tilde{f}_{k}^{C}+\partial_{a} \tilde{f}_{k}^{A} \partial_{b} \tilde{f}_{k}^{B} \Gamma_{A B}^{C}\left(\tilde{f}_{k}\right)=\partial_{c} \tilde{f}_{k}^{C} \widetilde{\Gamma}_{a b}^{c}-\left(I I_{k}\right)_{a b}^{C}
$$

where $\widetilde{\Gamma}_{a b}^{c}$ are Christoffel's symbols of $\tilde{g}_{k}$ and $I I_{k}\left(\partial_{a}, \partial_{b}\right)=\left(I I_{k}\right)_{a b}^{C} \partial_{C}$. By assumption,

$$
\left|\left(I I_{k}\right)_{a b}^{C}\right| \leq C_{0} \Lambda \sum_{A}\left|\partial_{a} \tilde{f}_{k}^{A} \partial_{b} \tilde{f}_{k}^{A}\right|
$$

where $C_{0}$ is a constant independent of $k$. Thus one obtains a uniform $C^{0}$ bound on $\partial_{a} \partial_{b} \tilde{f}_{k}^{C}$. Since $\bigcup_{k} \tilde{f}_{k}\left(M_{0}\right)$ is contained in a compact subset in $\widetilde{M}$, one concludes that $\tilde{f}_{k}$ subconverges to a $C^{1, \alpha}$ map $f_{0}$ in the $C^{1, \alpha^{\prime}}$ topology $\left(\alpha^{\prime}<\alpha\right)$. Clearly, $f_{0}^{*} \tilde{g}=g_{0}$, which implies $f_{0}:\left(M_{0}, g_{0}\right) \rightarrow(\widetilde{M}, \tilde{g})$ is an isometric immersion.

Remark. Without much difficulty, one can also show that if $(M, f)$ is almost totally geodesic in a closed Riemannian $m$-manifold $(\widetilde{M}, \tilde{g})$, that is $\operatorname{vol}(f) \leq$ $V$ and $\left|I I_{f}\right| \leq \varepsilon(n, V, \widetilde{M})$, then there is a $C^{2, \alpha}$ immersion $\hat{f}: M \rightarrow \widetilde{M}$ such that $\hat{f}$ is totally geodesic. One can prove this fact by a limit argument. See [JK] for the regularity of the limit immersion.

\section{IRREDUCIBLE HOMOGENEOUS MANIFOLDS}

A connected Riemannian manifold $(M, g)$ is said to be (isotropy) irreducible if for each point $p \in M$ the isotropy group $I_{p}$ acts irreducibly on $T_{p} M$ via its isotropy representation. $(M, g)$ must be a homogeneous space. Hence we call it an irreducible homogeneous Riemannian manifold. It is known that $g$ must be an Einstein metric. Further, by a theorem of Takahashi [T], any compact irreducible homogeneous Riemannian manifold can be isometrically minimally immersed into some $S^{K}(r) \subset \mathbb{R}^{K+1}$ using an orthonormal basis for an arbitrary eigenspace. See [L1, L2] for further discussion. 
Proof of Proposition 1. Let $(M, g)$ be a closed irreducible homogeneous Riemannian $n$-manifold satisfying (6). Let $\lambda_{1}$ denote the first nonzero eigenvalue and $E_{\lambda_{1}}$ the corresponding eigenspace. It is a finite-dimensional Hilbert space with $L^{2}$-norm. Take any orthonormal basis $\phi_{1}, \ldots, \phi_{K+1}$ for $E_{\lambda_{1}}$. Then for some constant $\alpha>0, f=\alpha \cdot\left(\phi_{1}, \ldots, \phi_{K+1}\right)$ is an isometric minimal immersion into $S^{K}(r) \subset \mathbb{R}^{K+1}$ with $r^{2}=n / \lambda_{1}$.

Regard $(M, f)$ as a submanifold in $\mathbb{R}^{K+1}$. Let $I I_{f}$ denote the normal curvature of $f$. Then $\operatorname{tr} I I_{f}:=\sum_{i=1}^{n} I I_{f}\left(e_{i}, e_{i}\right)=\frac{n}{r} \eta$, where $\eta$ denotes the unit normal vector field on $S^{K}(r) \subset \mathbb{R}^{K+1}$. By the Gauss equation,

$$
\operatorname{scal}(M)=n \lambda_{1}-\sum_{i=1}^{n}\left|I I_{f}\left(e_{i}, e_{i}\right)\right|^{2} .
$$

Thus

$$
\left|I I_{f}\right| \leq \sqrt{n \lambda_{1}+\lambda} .
$$

Notice that $(M, g)$ is Einstein and diam $=1$. It follows from [Che] that

$$
\lambda_{1} \leq c_{1}(n) \lambda+c_{2}(n) .
$$

Thus

$$
\left|I I_{f}\right| \leq C_{1}(n, \lambda) .
$$

It follows from (12), (13), and the Gauss equation that

$$
|\sec (M)| \leq C_{2}(n, \lambda) .
$$

It follows from Proposition 3 that

$$
\operatorname{inj}(M) \geq C_{3}(n, \lambda)>0 .
$$

Therefore, $\mathscr{H}(n, \lambda)$ is precompact in the $C^{\infty}$ topology, since all manifolds in $\mathscr{H}(n, \lambda)$ are Einstein.

\section{REFERENCES}

[AL] L. Andersson, The Pogorelov-Klingenberg theorem for submanifolds with bounded normal curvature, Report UMINF-87-80, Univ. of UMEA, 1980.

[AM] M. Anderson, Convergence and rigidity of manifolds under Ricci curvature bounds, Invent. Math. 102 (1990), 429-445.

[CGT] J. Cheeger, M. Gromov, and M. Tayler, Finite propagation speed, kernel estimates for functions of the Laplace operator, and the geometry of complete Riemannian manifolds, J. Differential Geom. 17 (1982), 15-53.

[Ch] J. Cheeger, Finiteness theorems for Riemannian manifolds, Amer. J. Math. 96 (1970), 61-74.

[Che] S. Y. Cheng, Eigenvalue comparison theorems and its geometry applications, Math. Z. 143 (1975), 289-297.

[G1] M. Gromov, Structures metriques pour les varietes Riemanniennes, Cedic-Fernand Nathan, 1981.

[G2] _ Pseudo holomorphic curves in symplectic manifolds, Invent. Math. 82 (1985), 307-347.

[GW] R. Greene and H. Wu, Lipschitz convergence of Riemannian manifolds, Pacific J. Math. 131 (1988), 119-141.

[H] R. Howard, Private communication.

[HS] D. Hoffman and J. Spruck, Sobolev and isoperimetric inequalities for Riemannian submanifolds, Comm. Pure Appl. Math. 27 (1974), 715-727. 
[JK] J. Jost and H. Karcher, Geometrische Methoden zur Gewinnung von a-priori-Schranken für harmonische Abbildungen, Manuscripta Math. 40 (1982), 27-77.

[K] A. Kasue, $A$ convergence theorem for Riemannian manifolds and some applications, Nagoya Math. J. 114 (1989), 21-51.

[L1] P. Li, Eigenvalue estimates on homogeneous manifolds, Comment. Math. Helv. 55 (1980), 347-463.

[L2] _ Minimal immersions of compact irreducible homogeneous Riemannian manifolds, $\mathbf{J}$. Differential Geom. 16 (1981), 105-115.

[MS] J. Michael and L. Simon, Sobolev and mean-value inequalities on generalized submanifolds of $\mathbb{R}^{n}$, Comm. Pure Appl. Math. 26 (1973), 361-379.

[N1] I. G. Nikolaev, Parallel translation and smoothness of the metric of spaces of bounded curvature, Soviet Math. Dokl. 21 (1980), 263-265.

[N2] Smoothness of the metric of spaces with bilaterally bounded curvature in the space of A. D. Aleksandrov, Siberian Math. J. 24 (1983), 247-263.

[Ps] S. Peters, Convergence of Riemannian manifolds, Compositio Math. 62 (1987), 3-16.

[PW] T. Parker and J. Wolfson, A compactness theorem for Gromov's moduli space, preprint, 1991.

[T] T. Takahashi, Minimal immersions of Riemannian manifolds, J. Math. Soc. Japan 18 (1966), 380-385.

[Y] R. Ye, Gromov's compactness theorem for pseudo-holomorphic curves, preprint, 1991.

DEPARTMENT OF MATHEMATICS, INDIANA UNIVERSITY-PURdue UNIVERSITY at INDIANAPOLIS, INDIANAPOLIS, INDIANA 46202-3216

E-mail address: zshen@math.iupui.edu 